\title{
The different path of T790M-positive EGFR-mutant lung cancer
}

\author{
Shinkyo Yoon ${ }^{1}$, Chang Min Choi ${ }^{1,2}$, Jae Cheol Lee $^{1}$ \\ ${ }^{1}$ Department of Oncology, ${ }^{2}$ Department of Pulmonary and Critical Care Medicine, University of Ulsan College of Medicine, Asan Medical Center, \\ Songpa-gu, Seoul, Korea \\ Correspondence to: Jae Cheol Lee, MD, PhD. Department of Oncology, University of Ulsan College of Medicine, Asan Medical Center, 88 Olympic- \\ ro, 43-gil, Songpa-gu, Seoul 05505, Korea. Email: jclee@amc.seoul.kr. \\ Comment on: Gaut D, Sim MS, Yue Y, et al. Clinical Implications of the T790M Mutation in Disease Characteristics and Treatment Response in \\ Patients With Epidermal Growth Factor Receptor (EGFR)-Mutated Non-Small-Cell Lung Cancer (NSCLC). Clin Lung Cancer 2018;19:e19-28.
}

Submitted Sep 19, 2018. Accepted for publication Sep 30, 2018.

doi: $10.21037 /$ atm.2018.10.01

View this article at: http://dx.doi.org/10.21037/atm.2018.10.01

Approximately half of the cases of acquired resistance to epidermal growth factor receptor-tyrosine kinase inhibitors (EGFR-TKIs) are mediated by the secondary T790M mutation $(1,2)$. Distinct clinical course of patients with T790M-positive lung cancer has been suggested, mostly in the context of more indolent progression and favorable prognosis $(3,4)$. It is in line with the pre-clinical study showing that EGFR-mutant cell lines with acquired T790M mutation exhibit slower growth although the way how the additional T790M mutation can affect the growth rate of EGFR-mutant lung cancer cells remains unclear (5).

Gaut et al. compared the clinical characteristics of patients with T790M-mediated resistance and the others after $1^{\text {st }}$-line EGFR-TKI. The group with T790M-positive lung cancer had a longer progression-free survival (PFS) on $1^{\text {st }}$-line EGFR-TKI (12.0 vs. 9.0 months, $\left.\mathrm{P}=0.12\right)$ despite the similar overall response rate. In addition, they showed a longer PFS on initial chemotherapy (5.0 vs. 4.0 months, $\mathrm{P}=0.025)$ while there was no difference in PFS on TKI rechallenge (4.0 vs. 3.0 months, $\mathrm{P}=0.94)$ (6).

$\mathrm{Li}$ et al. demonstrated that patients with $\mathrm{T} 790 \mathrm{M}$ mutation had significantly longer PFS (6.3 vs. 2.6 months, $\mathrm{P}=0.002$ ) and overall survival (39.8 vs. 23.2 months, $\mathrm{P}=0.044$ ) than those without T790M by the continuation of EGFR-TKI beyond progression (4). We can think the benefit of EGFRTKI after progression in two aspects. First, we acknowledge that double mutant lung cancer with a sensitizing mutation such as 19 deletion or L858R plus T790M is still dependent on EGFR signaling given that osimertinib, a $3^{\text {rd }}$ generation EGFR-TKI can effectively control this form of lung cancer by the suppression of EGFR signal (7). The proportion of T790M differs according to the degree of resistance indicating that T790M positivity itself cannot directly link to non-response to EGFR-TKI and there would be some in which EGFR-TKI is still effective if the proportion of T790M does not reach the certain level enough to bring the actual resistance despite the positive result of T790M $(8,9)$. Next, as authors indicated, discontinuation of EGFR-TKI can result in re-growth of TKI-sensitive clones having the rapid growing potential compared to T790M containing clones (10).

Then, does not this phenomenon apply to TKI rechallenge as it could not significantly prolong PFS in the studies of Gaut $e t$ al. Although it is possible that the intervening effect of combined drugs or drug-free holidays in TKI rechallenge brought the negative impact, it is more plausible to presume that the effect of continuation of EGFR-TKI and rechallenge is not so different in T790M-mediated resistance with persistent dependence to EGFR. It is more likely that the sample size was too small to prove the beneficial effect since we can observe the tendency of higher response rate to TKI rechallenge in T790M-positive patients.

However, the clinical implication of the continued EGFR-TKI beyond progression or rechallenge of $1^{\text {st }}$-line EGFR-TKI became less important since the emergence of osimertinib because switching to osimertinib in the wake of T790M is the best choice at present although those strategies can be still working in T790M-negative patients. The remaining issue on this regard is whether they can be applied to osimertinib-resistance or not, especially when osimertinib is being more popularly used as $1^{\text {st }}$-line therapy (11).

A retrospective study revealed that frontline EGFR- 
TKI significantly reduced the sensitivity of subsequent chemotherapy, which led to worse overall survival of this group compared with the control group receiving first-line chemotherapy followed by second-line EGFR-TKIs (12). Further, Gaut et al. showed that improved PFS on TKI if TKI was given after chemotherapy $(\mathrm{P}=0.007)$. OS has likewise been shown to be longer for patients receiving frontline versus post-TKI chemotherapy. It contradicts our current concept because we obviously prescribe EGFRTKI as $1^{\text {st }}$-line therapy in patients with EGFR-mutant lung cancer and we think it's the best for patients because the most effective therapy should come first. However, we have to admit that there is no well-performed comparison study about the efficacy between $1^{\text {st }}$ - and $2^{\text {nd }}$-line EGFR-TKI for EGFR-mutant lung cancer whereas we could see many clinical trials which compared EGFR-TKI and cytotoxic chemotherapy as $1^{\text {st }}$-line therapy. Nevertheless, we seem to doubt the necessity of launching the well-designed, randomized study from now on comparing the $1^{\text {st }}$ - and $2^{\text {nd }}$ line EGFR-TKI therapy for EGFR-mutant lung cancer unless more accumulated date would urge to do that.

\section{Acknowledgements}

None.

\section{Footnote}

Conflicts of Interest: The authors have no conflicts of interest to declare.

\section{References}

1. Chang YS, Choi CM, Lee JC. Mechanisms of Epidermal Growth Factor Receptor Tyrosine Kinase Inhibitor Resistance and Strategies to Overcome Resistance in Lung Adenocarcinoma. Tuberc Respir Dis (Seoul) 2016;79:248-56.

2. Rotow J, Bivona TG. Understanding and targeting resistance mechanisms in NSCLC. Nat Rev Cancer 2017;17:637-58.

Cite this article as: Yoon S, Choi CM, Lee JC. The different path of T790M-positive EGFR-mutant lung cancer. Ann Transl Med 2018;6(Suppl 1):S47. doi: 10.21037/atm.2018.10.01
3. Oxnard GR, Arcila ME, Sima CS, et al. Acquired resistance to EGFR tyrosine kinase inhibitors in EGFRmutant lung cancer: distinct natural history of patients with tumors harboring the T790M mutation. Clin Cancer Res 2011;17:1616-22.

4. Li W, Ren S, Li J, et al. T790M mutation is associated with better efficacy of treatment beyond progression with EGFR-TKI in advanced NSCLC patients. Lung Cancer 2014;84:295-300.

5. Chmielecki J, Foo J, Oxnard GR, et al. Optimization of dosing for EGFR-mutant non-small cell lung cancer with evolutionary cancer modeling. Sci Transl Med 2011;3:90ra59.

6. Gaut D, Sim MS, Yue Y, et al. Clinical Implications of the T790M Mutation in Disease Characteristics and Treatment Response in Patients With Epidermal Growth Factor Receptor (EGFR)-Mutated Non-Small-Cell Lung Cancer (NSCLC). Clin Lung Cancer 2018;19:e19-28.

7. Skoulidis F, Papadimitrakopoulou VA. Targeting the Gatekeeper: Osimertinib in EGFR T790M MutationPositive Non-Small Cell Lung Cancer. Clin Cancer Res 2017;23:618-22.

8. Ercan D, Zejnullahu K, Yonesaka K, et al. Amplification of EGFR T790M causes resistance to an irreversible EGFR inhibitor. Oncogene 2010;29:2346-56.

9. Park JH, Choi YJ, Kim SY, et al. Activation of the IGF1R pathway potentially mediates acquired resistance to mutant-selective 3rd-generation EGF receptor tyrosine kinase inhibitors in advanced non-small cell lung cancer. Oncotarget 2016;7:22005-15.

10. Lee JC, Jang SH, Lee KY, et al. Treatment of Non-small Cell Lung Carcinoma after Failure of Epidermal Growth Factor Receptor Tyrosine Kinase Inhibitor. Cancer Res Treat 2013;45:79-85.

11. Soria JC, Ohe Y, Vansteenkiste J, et al. Osimertinib in Untreated EGFR-Mutated Advanced Non-Small-Cell Lung Cancer. N Engl J Med 2018;378:113-25.

12. Zeng Z, Yan HH, Zhang XC, et al. Reduced chemotherapy sensitivity in EGFR-mutant lung cancer patient with frontline EGFR tyrosine kinase inhibitor. Lung Cancer 2014;86:219-24. 